PROCEEDINGS OF THE AMERICAN MATHEMATICAL SOCIETY

Volume 125, Number 5, May 1997, Pages 1367-1370

S 0002-9939(97)03722-2

\title{
BILOCAL DERIVATIONS OF STANDARD OPERATOR ALGEBRAS
}

\author{
JUN ZHU AND CHANGPING XIONG
}

(Communicated by Palle E. T. Jorgensen)

\begin{abstract}
In this paper, we shall show the following two results: (1) Let $A$ be a standard operator algebra with $I$, if $\Phi$ is a linear mapping on $A$ which satisfies that $\Phi(T)$ maps $\operatorname{ker} T$ into $\operatorname{ran} T$ for all $T \in A$, then $\Phi$ is of the form $\Phi(T)=T A+B T$ for some $A, B$ in $B(X)$. (2) Let $X$ be a Hilbert space, if $\Phi$ is a norm-continuous linear mapping on $B(X)$ which satisfies that $\Phi(P)$ maps ker $P$ into ran $P$ for all self-adjoint projection $P$ in $B(X)$, then $\Phi$ is of the form $\Phi(T)=T A+B T$ for some $A, B$ in $B(X)$.
\end{abstract}

In what follows $X$ stands for a Banach space (or Hilbert space) and $X^{*}$ for its norm dual. We denote by $(x, f)$ the duality pairing between elements $f \in X^{*}$ and $x \in X$, and we use the symbols " $B(X)$ ", " $L(X)$ ", " $F(X)$ ", " $I$ " and " $x \otimes f$ " to denote the set of all linear bounded operators on $X$, the set of all linear mappings on $X$, the set of all finite rank operators on $X$, the identity operator and the rank one operator $(*, f) x$ on $X$, respectively.

If $A$ is a Banach algebra, and $A_{1}$ is a Banach subalgebra of $A$, we say that a linear mapping $\Phi: A_{1} \rightarrow A$ is a derivation if $\Phi(a b)=\Phi(a) b+a \Phi(b)$ for any $a$ and $b$ in $A_{1}$. The derivation $\Phi$ is called inner if there exists an element $a$ in $A$ such that $\Phi(b)=b a-a b$ for any $b$ in $A_{1}$. We say that a linear mapping $\Phi: A_{1} \rightarrow A$ is a local derivation if for every $a$ in $A_{1}$, there exists a derivation $\delta_{a}: A_{1} \rightarrow A$, depending on $a$, such that $\Phi(a)=\delta_{a}(a)$. A linear mapping $\Phi$ is called a Jordan derivation if $\Phi\left(a^{2}\right)=a \Phi(a)+a \Phi(a)$ for every $a$ in $A_{1}$. We give the notion of bilocal derivation as follows:

Definition 1. If $A$ is a Banach subalgebra of $B(X)$, a linear mapping $\Phi: A \rightarrow$ $B(X)$ is called a bilocal derivation if for every $T$ in $A$ and $u$ in $X$, there exists a derivation $\delta_{T, u}: A \rightarrow B(X)$, depending on $T$ and $u$, such that $\Phi(T) u=\delta_{T, u}(T) u$.

Definition 2. Let $X$ be a Banach space, a Banach subalgebra $A$ of $B(X)$ is called a standard operator algebra if $A$ contains $F(X)$.

D. R. Larson and A. R. Sourour [5] have proved that every local derivation on $B(X)$ is a derivation. R. Kadison [4] and M. Brešar [1] have discussed normcontinuous local derivations on von Neumann algebras. It is obvious that every

Received by the editors June 14, 1995 and, in revised form, November 8, 1995.

1991 Mathematics Subject Classification. Primary 47D30, 47D25, 47B47.

Key words and phrases. Jordan derivation, standard operator algebra, bilocal derivation, local derivation.

Project supported by the Science Foundation of HBEC, People's Republic of China.

(C) 1997 American Mathematical Society 
local derivation is bilocal. In this paper, we shall prove that every bilocal derivation $\Phi: A \rightarrow B(X)$ is a derivation, where $A$ is a standard operator algebra.

We now prove the main theorems of this paper.

Theorem 3. Let $A$ with $I$ be a standard operator subalgebra of $B(X)$, and let $\Phi$ be a linear mapping from $A$ into $B(X)$, then the following properties hold:

(I) If $\Phi(T)$ maps $\operatorname{ker} T$ into $\operatorname{ran} T$ for all $T$ in $A$, then there exist linear operators $A$ and $B$ in $B(X)$ such that

$$
\Phi(T)=T A+B T, \text { for any } T \text { in } A .
$$

(II) If $\Phi$ is a bilocal derivation, then $\Phi$ is an inner derivation.

Proof. (I) Fix $f \in X^{*}$. By the condition (I) of the theorem, $\Phi(x \otimes f)$ maps ker $f$ into span $\{x\}$, so there exists a continuous linear functional $\lambda_{f, x}$ on ker $f$ such that

$$
\Phi(x \otimes f)(u)=\left(u, \lambda_{f, x}\right) x, \quad \text { for any } u \text { in } \operatorname{ker} f .
$$

For any $y \in X$, we have

$$
\Phi((x+y) \otimes f)(u)=\left(u, \lambda_{f, x+y}\right)(x+y) .
$$

On the other hand,

$$
\begin{aligned}
\Phi((x+y) \otimes f)(u) & =\Phi(x \otimes f)(u)+\Phi(y \otimes f)(u) \\
& =\left(u, \lambda_{f, x}\right) x+\left(u, \lambda_{f, y}\right) y .
\end{aligned}
$$

By comparing (2) and (3), we see that

$$
\left(u, \lambda_{f, x+y}-\lambda_{f, x}\right) x+\left(u, \lambda_{f, x+y}-\lambda_{f, y}\right) y=0 .
$$

Thus we obtain that $\lambda_{f, x+y}=\lambda_{f, x}=\lambda_{f, y}$, so $\lambda_{f, x}$ is independent of $x$. We write $\lambda_{f}=\lambda_{f, x}$. Let $g_{f}$ be a continuous linear extension of $\lambda_{f}$ to $X$. For any $u \in X-\operatorname{ker} f$, we define an operator $B_{u, f}$ as follows:

$$
B_{u, f} x=(u, f)^{-1}\left(\Phi(x \otimes f)(u)-\left(u, g_{f}\right) x\right) .
$$

It is obvious that $B_{u, f} \in L(X)$, thus we see that

$$
\Phi(x \otimes f)(u)=\left(u, g_{f}\right) x+(u, f) B_{u, f} x, \quad \text { for any } x \in X .
$$

Take any $v \in X-\operatorname{ker} f$ with $v \neq-u, v \neq 0$. Thus we have

$$
\Phi(x \otimes f)(u+v)=\left(u+v, g_{f}\right) x+(u+v, f) B_{u+v, f} x, \quad \text { for any } x \in X .
$$

The equalities (4), (5) and (6) yield $B_{u, f}=B_{v, f}$. Thus we may take $B_{u, f}=B_{f}$. It follows that

$$
\Phi(x \otimes f)(u)=\left(u, g_{f}\right) x+(u, f) B_{f} x, \quad \text { for any } u \text { in } X .
$$

Given any $u \in X$, we now fix a functional $f_{0} \in X^{*}$ with $\left(u, f_{0}\right)=1$. Thus we may take $g_{f_{0}} \in X^{*}$ and $B_{f_{0}} \in L(X)$ such that

$$
\Phi\left(x \otimes f_{0}\right)(u)=\left(u, g_{f_{0}}\right) x+\left(u, f_{0}\right) B_{f_{0}} x, \quad \text { for any } x \in X .
$$

We may take $B=B_{f_{0}}$. For any $f \in X^{*}$, we write $f_{1}=(u, f) f_{0}, f_{2}=f-f_{1}$. It is clear that $\left(u, f_{2}\right)=0$. Thus there exists a functional $g_{f_{2}}$ in $X^{*}$ and $B_{f_{2}}$ in $L(X)$ such that

$$
\Phi\left(x \otimes f_{2}\right)(u)=\left(u, g_{f_{2}}\right) x+\left(u, f_{2}\right) B_{f_{2}} x=\left(u, g_{f_{2}}\right) x,
$$


for any $x \in X$. If we take $b_{u, f}=(u, f) g_{f_{0}}+g_{f_{2}}$, then we have

$$
\begin{aligned}
\Phi(x \otimes f)(u) & =\left(\Phi\left(x \otimes(u, f) f_{0}\right)+\Phi\left(x \otimes f_{2}\right)\right)(u) \\
& =\left(u,(u, f) g_{f_{0}}+g_{f_{2}}\right) x+\left(u,(u, f) f_{0}\right) B_{f_{0}} x \\
& =\left(x \otimes b_{u, f}+B x \otimes f\right)(u) .
\end{aligned}
$$

We define a mapping $b_{f}$ by $b_{f}(u)=\left(u, b_{u, f}\right) x$. Note that $b_{f}(u)=\left(u, b_{u, f}\right) x=$ $\Phi(x \otimes f)(u)-(u, f) B x$ for any $x$ in $X$, so $b_{f}$ is a linear functional, and $b_{f}$ belongs to $X^{*}$. It follows that

$$
\Phi(x \otimes f)(u)=\left(x \otimes b_{f}+B x \otimes f\right)(u),
$$

for any $u \in X$. Thus we may define an operator $C$ by $C f=b_{f}$ for any $f \in X^{*}$. We claim that $B \in B(X)$ and $C=(\Phi(I)-B)^{*} \in B\left(X^{*}\right)$. In fact, for any $f \in X^{*}$ and $x \in X$ with $(x, f)=1$, if we write $P=x \otimes f$, and note that $\Phi(I-P)$ maps $\operatorname{ker}(I-P)(=\operatorname{span}\{x\})$ into $\operatorname{ran}(I-P)(=\operatorname{ker} f)$, then we have $P \Phi(I-P) P=0$, so $P \Phi(P) P=P \Phi(I) P$. Using the above equality, we can prove $B \in B(X)$ and $C=(\Phi(I)-B)^{*}$ by imitating the proof of Lemma 5 in [5]. Setting $A=\Phi(I)-B$, thus we have

$$
\Phi(x \otimes f)=(x \otimes f) A+B(x \otimes f) .
$$

Using the same proof as Theorem 1.2 in [5, p. 192], we obtain the desired conclusion.

(II) Since $\Phi$ is a bilocal derivation from $A$ into $B(X)$, and every derivation from $A$ into $B(X)$ is inner by [3], $\Phi$ satisfies the condition (I) of Theorem 3. Thus there are operators $A$ and $B$ in $B(X)$ such that equality (1) holds, and $A+B=\Phi(I)=0$, i.e. $B=-A$, so $\Phi$ is an inner derivation. The proof is complete.

Theorem 4. Let $X$ be a Hilbert space. If $\Phi$ is a norm-continuous linear mapping on $B(X)$ which satisfies that $\Phi(P)$ maps $\operatorname{ker} P$ into $\operatorname{ran} P$ for all self-adjoint projection $P$ in $B(X)$, then there are $A$ and $B$ in $B(X)$ such that

$$
\Phi(T)=T A+B T, \quad \text { for any } T \in B(X) .
$$

Proof. Let $P, Q$ be orthogonal self-adjoint projection in $B(X)$. By the condition of the theorem, we know that $\Phi(P)$ maps ker $P$ into ran $P$. Thus we have

$$
(I-P) \Phi(P)(I-P)=0 .
$$

It follows that $\Phi(P)=\Phi(P) P+P \Phi(P)-P \Phi(P) P$. Substituting $I-P$ for $P$ in the equality (10), we have $P \Phi(I-P) P=0$, i.e. $P \Phi(P) P=P \Phi(I) P$. Thus we obtain

$$
\Phi(P)=\Phi(P) P+P \Phi(P)-P \Phi(I) P .
$$

According to the equality (11), we then have

$$
\begin{aligned}
\Phi(P)+ & \Phi(Q)=\Phi(P+Q) \\
= & \Phi(P+Q)(P+Q)+(P+Q) \Phi(P+Q)-(P+Q) \Phi(P+Q)(P+Q) \\
= & (\Phi(P) P+P \Phi(P)-P \Phi(I) P)+(\Phi(Q) Q+Q \Phi(Q)-Q \Phi(I) Q) \\
& +\Phi(P) Q+\Phi(Q) P+P \Phi(Q)+Q \Phi(P)-Q \Phi(I) P-P \Phi(I) Q .
\end{aligned}
$$

Consequently

$$
\Phi(P) Q+\Phi(Q) P+P \Phi(Q)+Q \Phi(P)-Q \Phi(I) P-P \Phi(I) Q=0 .
$$


Using the above equality (12), by the same proof as Lemma 1 in [1], we may prove that

$$
\Phi\left(a^{2}\right)=\Phi(a) a+a \Phi(a)-a \Phi(I) a, \quad \text { for any } a \text { in } B(X) .
$$

We claim that $\Phi(x \otimes y)$ maps $\operatorname{ker}(x \otimes y)$ into $\operatorname{ran}(x \otimes y)$ for every $x, y \in X$. In fact, if $(x, y) \neq 0$, then we have

$$
\begin{aligned}
\Phi(x \otimes y) & =(x, y)^{-1} \Phi\left((x \otimes y)^{2}\right) \\
& =(x, y)^{-1}\{\Phi(x \otimes y)(x \otimes y)+(x \otimes y) \Phi(x \otimes y)-(x \otimes y) \Phi(I)(x \otimes y)\} .
\end{aligned}
$$

It follows that $\Phi(x \otimes y)$ maps $\operatorname{ker}(x \otimes y)$ into $\operatorname{span}\{x\}$. If $(x, y)=0$, without loss of generality, we may assume that $\operatorname{dim} X>2$ and $x \neq 0, y \neq 0$. Take $y_{1}$ and $y_{2}$ in $X$ with $\left(x, y_{i}\right)=1$ and $y_{2} \in\left\{y, y_{1}\right\}^{\perp}$. It is obvious that $\left\{y, y_{1}\right\}^{\perp} \vee\left\{y, y_{2}\right\}^{\perp}=\{y\}^{\perp}=$ $\operatorname{ker}(x \otimes y)$. Since $\left(x, y_{i}\right)=\left(x, y+y_{i}\right)=1 \neq 0$, and $\Phi(x \otimes y)=\Phi\left(x \otimes\left(y+y_{i}\right)\right)-\Phi\left(x \otimes y_{i}\right)$ $(i=1,2), \Phi(x \otimes y)$ maps $\left\{y+y_{i}\right\}^{\perp} \wedge\left\{y_{i}\right\}^{\perp}\left(=\left\{y, y_{i}\right\}^{\perp}\right)$ into $\operatorname{span}\{x\}$. It follows that $\Phi(x \otimes y)$ maps $\left\{y, y_{1}\right\}^{\perp} \vee\left\{y, y_{2}\right\}^{\perp}(=\operatorname{ker}(x \otimes y))$ into $\operatorname{span}\{x\}$.

We may prove that there are $A_{1}$ and $B_{1}$ in $B(X)$ such that $\Phi(x \otimes y)=$ $(x \otimes y) A_{1}+B_{1}(x \otimes y)$ by imitating the proof of Theorem 3 . Thus we have

$$
\Phi(F)=F A_{1}+B_{1} F, \quad \text { for any } F \text { in } F(X) .
$$

Let $\Psi$ be defined by $\Psi(T)=T A_{1}+B_{1} T$ for every $T$ in $B(X)$, and let $h=\Phi-\Psi$. It is obvious that $h$ satisfies the hypotheses of $\Phi$ in the statement of Theorem 4, and $h(F)=0$ for any $F$ in $F(X)$. Using the equalities (13), we have $F h(I) F=0$ for every $F$ in $F(X)$, so $h(I)=0$. Using the equality (13) to $h$, we see that $h$ is a Jordan derivation. By Theorem 1 in [2], $h$ is a derivation. It follows by Theorem in [3] that $h$ is an inner derivation. Thus there are $A_{2}$ and $B_{2}$ in $B(X)$ such that $h(a)=a A_{2}+B_{2} a$ for every $a$ in $B(X)$. Take $A=A_{1}+A_{2}$ and $B=B_{1}+B_{2}$. Thus we obtain that

$$
\Phi(a)=h(a)+\Psi(a)=a\left(A_{1}+A_{2}\right)+\left(B_{1}+B_{2}\right) a=a A+B a,
$$

for any $a$ in $B(X)$. The proof is complete.

\section{ACKNOWLEDGEMENT}

We wish to express our thanks to Professor Shijie Lu and the referee for helpful suggestions.

\section{REFERENCES}

1. M. Brešar, Characterizations of derivations on some normed algebras with involution, J. Algebra 152 (1992), 454-462. MR 94e:46098

2. M. Brešar, Jordan derivation on semiprime rings, Proc. Amer. Math. Soc. (4) 104 (1988), 1003-1006. MR 89:16004

3. P. R. Chernoff, Representations, automorphism and derivation of some operator algebras, J. Funct. Anal. 12 (1973), 275-289. MR 50:2934

4. R. Kadison, Local derivation, J. Algebra 130 (1990), 494-509. MR 91f:46092

5. D. R. Larson and A. R. Sourour, Local derivation and local automorphism of $B(X)$, Proc. Sympos. Pure Math. (2) 51 (1990), 187-194. MR 91k:47106

6. P. Semrl, Additive derivation of some operator algebras, Illinois J. Math. (2) 35 (1991), 234240. MR 92b: 47068

Department of Mathematics, Hubei Institute for Nationalities, Enshi, Hubei, 445000, People's Republic of China 